\title{
El Origen De La Vida Y La Naturaleza Humana En Las Creencias De La Población En General
}

\author{
Dr. Jesús Silva Bautista \\ Lic. Venazir Herrera Escobar \\ Dr. Rodolfo Corona Miranda
}

Universidad Nacional Autónoma de México, Facultad de Estudios

Superiores Zaragoza. México

doi: 10.19044/esj.2016.v12n32p1 URL:http://dx.doi.org/10.19044/esj.2016.v12n32p1

\begin{abstract}
Different explanations that have been created around the origin of the universe, human nature and the role of human beings. These explanations have been founded primarily on religious, philosophical and scientific responses based on the belief system. Prior to this premise, it became necessary to investigate what were the beliefs concerning the origin of life and the nature of the human population. Taking into consideration this objective there was a selected sample of 600 people from the general population who answer the five-point Likert type survey. Through statistical tests and validated instrument, it performed comparisons between the beliefs of people according to their sex, educational background, age and marital status. The statistical results showed significant differences between beliefs about the origin of life and human nature in the general population in terms of gender, educational background, age and marital status. Based on the results, the role of beliefs on the behavior of people is basically characterized by how knowledge is acquired on the basis of their theory and practice. Through these means, they know each other's processes, scientific and nonscientific principles; and assisted on structuring their interaction with reality, the world, their peers and themselves.
\end{abstract}

Keywords: Belief, science, religion, action

\section{Resumen}

Las diferentes explicaciones que se han creado en torno al origen del universo, la naturaleza humana y el propio papel del ser en el mundo, han sido explicaciones que se fundamentan principalmente en respuestas religiosas, filosóficas y científicas; respuestas que tienen como base el sistema de creencias que el ser humano posee. Ante esto, surgió la necesidad 
de investigar ¿Cuáles son las creencias sobre el origen de la vida y naturaleza humana de población en general? Dado el objetivo de conocer este tipo de creencias se seleccionó una muestra de 600 personas de la población en general a quienes se les aplicó una encuesta con tipo de respuesta Likert de cinco puntos. A través de pruebas estadísticas se validó el instrumento y se realizó una comparación entre las creencias de las personas de acuerdo a su sexo, formación académica, edad y el estado civil. Los resultados muestran diferencias estadísticamente significativas entre las creencias sobre el origen de la vida y naturaleza humana de población en general en función de su sexo, formación académica, edad y el estado civil. Con base a los resultados, el papel que desempeñan las creencias en el comportamiento de las personas se caracteriza básicamente por el cómo se adquiere el conocimiento base de su teoría y práctica. A través de ellas se conocen los procesos y principios científicos y no científicos que estructuran su interacción con la realidad, con el mundo, con sus iguales y consigo mismo.

Palabras clave: Creencia, ciencia, religión, comportamiento

\section{Introducción}

Gran parte de las nociones que el hombre posee sobre el mundo son fruto de sus creencias; así lo señala García (2009) al plantear que éstas sirven como soporte en la medida en que son medios relativamente estables de recibir la realidad y las bases para poder articularla. En este sentido, las creencias son el eje que permite dar sentido a la realidad, la información que el sujeto conserve sobre el objeto no necesariamente tiene que ser verdadera para ser admitida como real. El sujeto puede guardar una imagen de la realidad sin tener fundamento científico alguno que justifique sus creencias; del mismo modo, puede enfrentar argumentos que resulten ser contrarios a su concepción, y aun así, continuar creyendo. Las creencias son aceptadas como algo real, en la medida que se fundan en la existencia de los hombres y sirven como fundamento de la vida.

Bajo este contexto, no importa de dónde provenga una creencia, sólo basta con que se de crédito a una afirmación para que ocupe un lugar en el sistema de creencias. El hombre está hecho para creer y necesita de ello para dar una continua explicación a lo que acontece a su alrededor. El contenido de sus creencias es elegido más o menos libremente en función de la satisfacción que le produzca (Díaz, 2004).

Denominar lo intangible es algo realmente complicado y subjetivo; por ello, en algunas ocasiones el concepto de creencia resulta difuso (Pajares, 1992), y a pesar de las numerosas investigaciones realizadas siguen existiendo cuestiones controvertidas en torno a su definición (Llinares, 
1995). Sin embargo, existe en la literatura diferentes autores que se han esforzado por definir concretamente qué son las creencias.

Para Fishbein (1967), la creencia representa la información que la persona tiene acerca del objeto, que está unido a algún atributo, es una hipótesis de probabilidad o improbabilidad en relación con la naturaleza del objeto y sus relaciones con otros objetos. Sobre esta línea, Fishbein y Ajzen (1975) sostienen:

"En términos generales, las creencias se refieren a los juicios de una persona de probabilidad subjetiva sobre algún aspecto discriminable de su mundo; que se ocupan de la comprensión de la persona misma y de su entorno. En concreto, se ha definido la creencia como la probabilidad subjetiva de la relación entre el objeto de la creencia y algún otro objeto, valor, concepto o atributo. Esta definición implica que la formación de la creencia involucra el establecimiento de un vínculo entre dos aspectos cualquiera del mundo del individuo” (p.131)

Definición similar es la que plantean Corral y Verdugo (2001, citados en, Rodríguez y Sotelo, 2007) al referirse a las creencias como “(...) tendencias a relacionar objetos, eventos o situaciones, empleando criterios convencionales o la experiencia previa como marco de referencia” (pp. 2325). En este sentido, las creencias no son actos específicos, sino tendencias a actuar; el aspecto relacional de esta variable refiere a la propensión de los sujetos a ligar o a unir causalmente a dos o más objetos o atributos. De modo que, las creencias además de ser un vínculo entre un objeto y un atributo con un cierto nivel de probabilidad, puede concebirse como, una condición inicial subjetiva que explica un conjunto de comportamientos aparentemente inconexos, y al entender la causa como condición inicial, la creencia es una causa del comportamiento. Así, creer implica tener una serie de expectativas formuladas a modo de hipótesis, que regulan las acciones y las relaciones del sujeto con el mundo en torno (Olson y Zanna, 1987; Villoro, 1996).

Al ser juicios y evaluaciones que las personas hacen de sí mismas y del mundo que las rodea, las creencias actúan como filtros a través de los cuales se integra la información proveniente del mundo interior y exterior, y contrario a lo que pudiera pensarse, no se derivan de una evidencia ambiental o conductual, sino que, la preceden y son las que le dan significado.

Durante la década de los noventa, Pajares (1992) realizó un estudio sobre creencias ostentadas por profesores, donde expresa que conceptualizar un sistema de creencias implica comprender que “este sistema está compuesto de creencias conectadas unas a otras, y a otras estructuras cognitivas/afectivas" (p. 325); asimismo, afirma que "la creencia se basa en la evaluación y en el juicio” De opinión similar, Llinares (1995) asume que "un aspecto importante es que las creencias no pueden ser directamente 
observadas o medidas, por lo tanto, se deben inferir de lo que las personas dicen, pretenden y hacen” (p.9).

En este contexto, Pajares (1992) plantea que las creencias representan las verdades personales indiscutibles, derivadas de la experiencia o fantasía, con un fuerte componente evaluativo y afectivo. Este autor, pone énfasis a la carga afectiva de las creencias, ya que, se encuentra relacionada con las preferencias, inclinaciones y líneas de acción del sujeto. Así, las creencias pueden mostrar aspectos afectivos en el individuo.

Las creencias son construcciones mentales de la experiencia; integradas en esquemas o conceptos que se mantienen como verdaderas, pero que pueden ser cuestionadas en el futuro; abarcan todas las cuestiones acerca de las cuales no se dispone de un conocimiento seguro, pero en las que se confía lo suficiente como para actuar de acuerdo con ellas, por ello, actúan como guías de la conducta (Dewy, 1984; Sigel, 1985, citados en, Latorre y Blanco, 2007). De acuerdo con esta proposición, Villoro (1996) mantiene que el sentimiento de convicción o de firmeza no puede definir la creencia y que por lo menos para actuar de acuerdo a ellas se debe tener una condición de disposición. Las creencias se forman tempranamente; se mantienen inmutables frente a las contradicciones causadas por la razón, el tiempo, la enseñanza o la experiencia; se adquieren a través de procesos de aprendizaje asociativo; pero también, tienen un origen cultural, en tanto se construyen en formatos de interacción social; son producto de la construcción del mundo; el ser humano las utiliza para tomar decisiones, recordar, interpretar, predecir y controlar sucesos. Se les puede ver como verdades personales incontrovertibles que son idiosincrásicas, con mucho valor afectivo y componentes evaluativos que residen en la memoria episódica (Nespor, 1987; Pajares, 1992; Solar y Díaz, 2009).

Una creencia constantemente inculcada en los primeros años de vida, cuando el cerebro es más impresionable, parece adquirir casi la naturaleza de un instinto (Darwin, 1987).

De idea similar es la de Díaz (2002, citado en, Rodríguez y Sotelo, 2007) cuando expresa que las creencias son un producto cultural, que constituye a una premisa sociocultural que respalda la cultura y sus fuerzas, permitiendo encontrar semejanzas en grupos sociales.

A diferencia de Pepitone (1991), Thompson (1992) afirma que las creencias “(...) no son consensuales, (...) son a menudo mantenidas o justificadas por razones que no cumplen estos criterios, y, por tanto, son caracterizadas por una falta de acuerdo sobre cómo tienen que ser evaluadas o juzgadas” (p. 129-130). Al respecto, Ponte (1994b) destaca que las creencias son proposiciones no demostradas y que “(...) el sistema de creencias no requiere de un consenso social respecto a su validez o 
propiedad. Las creencias personales no requieren, incluso, conciencia interna” (1994a, p. 5-6).

Las creencias propiamente dichas tienen un rango valorativo tan importante que se constituyen en una ética que justifica y enjuicia prácticas sociales. No se asumen por comprobación lógica, sino por conformidad; no se duda de ellas y para defenderlas se repite una argumentación aprendida mecánicamente; generan certidumbre psicológica y cuando se cuestionan se produce angustia; se dan por entendidas y se transmiten haciendo uso de signos (Arispe, 1989).

\section{Método}

\section{Participantes}

Para los fines de la investigación se eligió una muestra conformada por habitantes de la Zona Metropolitana. La muestra fue no probabilística intencional, por cuota de 600 personas pertenecientes a la población en general. De ella el 50\% son hombres y el 50\% restante mujeres. En lo que se refiere a la formación académica, el 14.2\% tienen como máximo nivel de estudios la educación primaria, el 10.8 \% la secundaria, el 18.3 \% cursaron hasta la preparatoria, el 31.5\% son licenciados y el 25.2\% cuentan con posgrado. En cuanto a la edad y el estado civil el 38.3\% son menores de 40 años, el $29.7 \%$ se encuentra entre los 40 y 49 años y el 32\% son mayores de 50 años; el $37.5 \%$ son solteros, el $50 \%$ casados y el $12.5 \%$ vive en unión libre.

\section{Instrumento}

El instrumento que se utilizó para medir las creencias sobre el Origen de la Vida y Naturaleza Humana está constituido por dos categorías de estudio y cada una dividida en tres subcategorías de análisis:

Categoría 1. Creencias hacia el Origen de la Vida. Se encuentra distribuido en tres categorías de análisis: Teoría de la Evolución de las Especies de Charles Darwin (Darwin, 2003), Creacionismo (Gould, 2000,2006) y la Teoría del Diseño Inteligente (Dembski, 2005, 2007).

Categoría 2. Creencias hacia la Naturaleza Humana. Está constituida por posturas como el Judeocristianismo (Pinker, 2003), el Materialismo Dialéctico de Marx y Engels (2011) y Fromm (1958, 1973).

A partir de estas dos categorías, se elaboró una escala Likert con 48 reactivos distribuidos en 24 por cada una y 8 reactivos por cada subcategoría, cada uno con cinco intervalos de respuesta: $1=$ Totalmente de acuerdo, $2=$ De acuerdo, 3= Ni de acuerdo, ni en desacuerdo, 4= En desacuerdo, 5= Totalmente en desacuerdo. 
Antes de ser aplicado a la muestra seleccionada, el instrumento fue sometido a diferentes pruebas de confiabilidad y validez.

\section{Resultados}

\section{Propiedades del instrumento}

En una primera aproximación se hizo un análisis descriptivo de los resultados de la puntuación en cada reactivo, considerando la distribución y la discriminación (Ver, Tabla1). Para el primer análisis se emplearon las respuestas obtenidas por la muestra evaluada, a través de las medias y las varianzas conseguidas. La media de respuesta a los reactivos se sitúa en 2.64; por otra parte, la varianza de los reactivos oscilan entre 2.16 (reactivo 1) y 3.38 (reactivo 18), obteniendo la varianza media de los reactivos un valor de 1.796. La capacidad discriminativa del instrumento se refleja en el índice de discriminación, este índice (correlación reactivo-total) refleja el grado de homogeneidad de los reactivos que componen la escala. En este caso, los valores del índice están comprendidos entre -.225 (reactivo 4) y .679 (reactivo 38). Finalmente en la última columna de la tabla 1 se presentan los valores del Alfa de Cronbach si se elimina el elemento.

Considerando todos los reactivos de la escala se calculó la confiabilidad encontrándose un índice de $\alpha=0.866$, lo cual indica que existe una consistencia interna entre los reactivos que la conforman. A pesar de las diferencias en la calidad de los reactivos que han sido puestas de relieve mediante los índices y estadísticos mencionados, se eliminaron aquellos reactivos cuyo índice fuera $\geq .20$ (reactivos 2, 3, 4 y 9). Después de la eliminación de los reactivos se volvió a calcular el coeficiente de Alfa de Cronbach de la escala total obteniéndose un valor de $\alpha=0.892$.

\begin{tabular}{c|c|c|c|c}
\hline \multicolumn{4}{c}{ Tabla 1. Media, varianza y estadística de relación reactivo - total en el instrumento de } \\
\multicolumn{5}{c}{ creencias sobre el Origen de la Vida y Naturaleza Humana } \\
\hline Reactivo & Media & Varianza & $\begin{array}{c}\text { Correlación elemento- } \\
\text { total corregida }\end{array}$ & $\begin{array}{c}\text { Alfa de Cronbach si se } \\
\text { elimina el elemento }\end{array}$ \\
\hline 1 & 2.16 & 1.459 & .286 & .867 \\
2 & 2.48 & 1.983 & -.138 & .872 \\
3 & 2.52 & 1.776 & .124 & .871 \\
4 & 2.87 & 2.529 & -.225 & .875 \\
5 & 2.85 & 2.203 & .555 & .858 \\
6 & 2.88 & 1.732 & .251 & .866 \\
7 & 2.33 & 1.832 & .299 & .871 \\
8 & 3.28 & 2.140 & .439 & .861 \\
9 & 2.55 & 1.714 & -.110 & .871 \\
10 & 2.46 & 1.898 & .602 & .858 \\
11 & 2.75 & 2.129 & .589 & .858 \\
12 & 2.73 & 1.621 & .246 & .869 \\
13 & 2.92 & 2.189 & .610 & .858 \\
14 & 3.05 & 2.148 & .556 & .859 \\
15 & 2.54 & 1.567 & .230 & .869
\end{tabular}




\begin{tabular}{|c|c|c|c|c|}
\hline 16 & 2.76 & 2.311 & .649 & .857 \\
\hline 17 & 2.75 & 2.339 & .602 & .858 \\
\hline 18 & 3.38 & 1.952 & .520 & .860 \\
\hline 19 & 2.25 & 1.318 & .259 & .866 \\
\hline 20 & 2.90 & 1.826 & .382 & .862 \\
\hline 21 & 2.79 & 2.296 & .649 & .857 \\
\hline 22 & 2.41 & 1.557 & .248 & .869 \\
\hline 23 & 3.03 & 2.141 & .594 & .858 \\
\hline 24 & 2.54 & 2.455 & .227 & .868 \\
\hline 25 & 2.30 & 1.222 & .260 & .864 \\
\hline 26 & 2.48 & 1.482 & .268 & .866 \\
\hline 27 & 2.84 & 2.257 & .656 & .857 \\
\hline 28 & 2.28 & 1.506 & .230 & .869 \\
\hline 29 & 2.90 & 2.317 & .561 & .858 \\
\hline 30 & 2.68 & 2.236 & .657 & .857 \\
\hline 31 & 2.45 & 1.240 & .228 & .866 \\
\hline 32 & 2.56 & 1.756 & .365 & .863 \\
\hline 33 & 2.75 & 1.385 & .288 & .865 \\
\hline 34 & 2.73 & 2.305 & .648 & .857 \\
\hline 35 & 2.68 & 1.783 & .543 & .859 \\
\hline 36 & 3.11 & 2.141 & .491 & .860 \\
\hline 37 & 2.49 & 1.369 & .290 & .867 \\
\hline 38 & 2.71 & 2.466 & 679 & .856 \\
\hline 39 & 2.10 & 1.098 & .295 & .865 \\
\hline 40 & 2.47 & 1.338 & .235 & .865 \\
\hline 41 & 2.45 & 1.249 & .200 & .867 \\
\hline 42 & 2.55 & 1.110 & .295 & .867 \\
\hline 43 & 2.19 & 1.128 & .205 & .866 \\
\hline 44 & 2.24 & 1.126 & .262 & .866 \\
\hline 45 & 2.98 & 1.921 & .591 & .858 \\
\hline 46 & 2.71 & 1.665 & .443 & .861 \\
\hline 47 & 3.15 & 2.306 & .459 & .861 \\
\hline 48 & 2.65 & 2.411 & .634 & .857 \\
\hline
\end{tabular}

Como resultado de estos análisis la escala quedo conformada por 44 reactivos a los cuales se les realizó un análisis factorial con rotación ortogonal con el fin de determinar si la agrupación de los reactivos corresponde a sus dimensiones teóricas, es decir, una correspondencia empírica respecto al marco teórico de la investigación y así reducir los datos a partir del agrupamiento de las variables que resultan homogéneas. A través de este análisis se encontró que la medida de adecuación muestral KaiserMeyer-Olkin fue alta $(0,970)$ y la prueba de esfericidad de Bartlett fue significativa al arrojar un valor aproximado para $946 \mathrm{gl} \mathrm{de} \mathrm{c}^{2}=20333.297(\mathrm{p}$ $=.000$ ). La comunalidad de cada reactivo indica valores mayores a 0.3 por lo que no se extrajeron reactivos de la escala.

Por medio del método de rotación de normalización Varimax con Káiser que convergió en 15 iteraciones y el método de análisis de componentes principales se extrajeron cuatro factores y se conservaron 
aquellos con valores propios por encima de 1 (criterio de Káiser), factores que en su conjunto explican el 58.832\% de la varianza total (Ver, Tabla 2). Con este procedimiento se eliminaron 3 reactivos, en todos los casos porque no alcanzaron el criterio de pesos factoriales iguales o superiores a 0.35 , o bien, compartieron peso factorial en otro factor. En la Tabla 3 se muestra la solución factorial obtenida.

Tabla 2. Varianza explicada, varianza acumulada, medias y desviaciones estándar de cada factor

\begin{tabular}{ccccc}
\hline Factores & $\begin{array}{c}\text { \% varianza } \\
\text { explicada }\end{array}$ & $\begin{array}{c}\% \\
\text { varianza } \\
\text { acumulada }\end{array}$ & M & DE \\
\hline $\begin{array}{c}\text { 1. Naturaleza Humana - Origen de la } \\
\text { Vida: Creencias Religiosas (NH-OV: CR). } \\
\text { Religión }\end{array}$ & 35.075 & 35.075 & 2.87 & 1.179 \\
\hline $\begin{array}{c}\text { 2. Naturaleza Humana: Creencias } \\
\text { Científicas Sociales (NH: CCS). } \\
\quad \text { Marxismo- Fromm }\end{array}$ & 10.420 & 45.495 & 1.61 & .553 \\
\hline $\begin{array}{c}\text { 3. Origen de la Vida: Creencias Científicas } \\
\text { Naturales (OV: CCN). Evolución }\end{array}$ & 8.623 & 54.118 & 2.41 & .943 \\
\hline $\begin{array}{c}\text { 4. Naturaleza Humana: Creencias } \\
\text { Científicas Sociales (NH: CCS). Fromm }\end{array}$ & 4.715 & 58.833 & 2.45 & .870 \\
\hline
\end{tabular}

Tabla 3. Matriz de componentes rotados, varianza total explicada y Alpha de Cronbach por factores Carga Factorial

Reactivo
Facto Facto Facto Facto r 1. r 2. r $3 . \quad$ r 4. $\mathrm{NH}-\mathrm{NH}: \mathrm{OV}: \mathrm{C} \mathrm{NH}$ OV: CCS CN CCS $\mathrm{CR}$

38. Dios creó al hombre a su imagen y semejanza.

21. Las personas debemos toda nuestra existencia al creador divino.

27. Creo que cada ser vivo que existe en la Tierra proviene de un acto de creación divina.

16. Para mí un ser divino creó la vida con un propósito determinado.

34. Para mí Dios es el principio y fin de todas las cosas.

30. Pienso que la naturaleza humana es un don divino.

17. Para mí Cristo determina la salvación humana.

48. Dios le brindo la libertad al ser humano.

13. Pienso que la complejidad de la diversidad de formas de vida en la Tierra implicó la intervención de un diseñador divino.

14. Considero que los cambios en el diseño biológico de los seres vivos son una respuesta planeada por un creador divino.

23. Creo que un Agente Divino fue quien permitió la evolución de los seres vivos.

11. Creo que el plan de Dios sobre la creación de la humanidad se encuentra plasmado en la Biblia.

5. La vida en la Tierra tuvo que ser el resultado de acciones divinas emprendidas de forma voluntaria por Dios.

\begin{tabular}{l|l|l|l}
.906 & -.083 & -.085 & -.083 \\
.900 & -.127 & -.073 & -.110 \\
.899 & -.122 & -.077 & -.098 \\
.888 & -.115 & -.086 & -.062 \\
.880 & -.133 & -.084 & -.060 \\
.877 & -.104 & -.079 & -.067 \\
.854 & -.148 & -.116 & -.050 \\
.850 & -.103 & -.081 & -.015 \\
.835 & -.100 & -.125 & -.011 \\
.827 & -.190 & -.156 & -.101 \\
.824 & -.125 & -.101 & -.058 \\
.815 & -.101 & -.184 & -.024 \\
.806 & -.133 & -.157 & -.012
\end{tabular}


29. El ser humano es la criatura más noble creada por Dios.

36. Pienso que la vida se convierte en una constante lucha cuando se desobedece a Dios.

45. Pienso que tras la diversidad de los seres vivos yace un diseñador inteligente.

47. La Biblia es la principal autoridad del conocimiento humano.

10. El hombre posee una naturaleza espiritual representada por Dios.

35. Creo que el ser humano es el producto de la existencia de un diseño divino preconcebido en la naturaleza.

8. Considero que la edad del universo puede estimarse basándose en la Biblia

46. Creo que para considerarse humano las personas deben ser bondadosas

20. A la naturaleza por sí sola le fue imposible crear las complejas estructuras que componen la vida.

18. La complejidad en las estructuras de los seres vivos es producto de la evolución.

41. Pienso que el ser humano es autocreador de su contexto vital.

44. Considero que el ser humano es el único responsable de su condición de vida.

43. Creo que las personas somos producto de nuestra sociedad.

37. A las personas se nos define a partir del momento en el vivimos.

40. La transmisión del conocimiento es lo que nos ha hecho humanos.

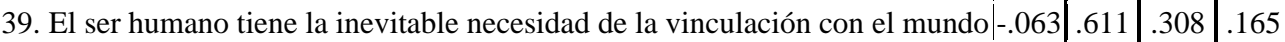

15. Lo único que interviene en el desarrollo de la Humanidad es la acción del hombre sobre ésta.

31. Creo que las necesidades del ser humano son únicamente producto de su sociedad.

24. Considero que los procesos socioculturales crean al Ser Humano.

22. La similitud biológica entre diferentes seres vivos permite reconstruir la relación entre las diferentes líneas evolutivas.

28. Los fósiles demuestran la evolución de los seres más sencillos hasta los más complejos.

26. Para mí uno de los productos más significativos de la evolución es el Ser Humano.

12. Considero que la Teoría de la Evolución es la mejor explicación sobre el surgimiento de la vida en la Tierra.

7. Pienso que la multitud de formas de vida que vemos hoy en día son el resultado del proceso de evolución.

19. Pienso que la naturaleza del hombre lo capacita para la búsqueda de la libertad.

25. Los seres humanos son una totalidad conformada por su familia y el resto de la sociedad.

1. Pienso que las relaciones sociales son la base sobre la que se crea el Ser Humano.

6. La cultura es la creadora del Ser Humano.

Total de reactivos

\begin{tabular}{c|c|c|c}
-.294 & .504 & .276 & .170 \\
-.153 & .470 & .223 & .407 \\
-.284 & .436 & .328 & .431 \\
-.345 & .282 & .698 & .040 \\
-.327 & .307 & .692 & .073 \\
-.097 & .225 & .625 & .142 \\
-.314 & .245 & .601 & .076 \\
-.396 & .176 & .575 & .351 \\
-.067 & .245 & .550 & .279 \\
.051 & .271 & .089 & .636 \\
-.169 & .124 & .322 & .632 \\
-.062 & .394 & .168 & .458 \\
23 & 9 & 6 & 3 \\
35.07 & 10.42 & 8.623 & 4.715 \\
5 & 0 & .851 & .529 \\
.975 & .861 & & \\
\hline
\end{tabular}

\% de Varianza Total Explicada Valor de coeficiente Alpha de Cronb 
El Factor 1 Naturaleza Humana y Origen de la Vida: Creencias Religiosas (NH-OV: CR). Religión está compuesto por reactivos cuyo contenido se fundamenta en los postulados del Creacionismo (Gould, 2000,2006), la Teoría del Diseño Inteligente (Dembski, 2005, 2007) y el Judeocristianismo (Pinker, 2003). Los reactivos de este factor están basados en la existencia de un ser superior, inteligente y con la voluntad de dirigir la creación y el proceso de evolución, así como el destino de todo ser vivo. Este factor se compone por 23 afirmaciones de las 41 totales. El mayor valor absoluto es de .906 correspondiente al ítem "Dios creó al hombre a su imagen y semejanza”. La varianza explicada de este factor corresponde a un $35.075 \%$ con un valor de coeficiente Alpha de Cronbach de $\alpha=0.975$.

El Factor 2 Naturaleza Humana: Creencias Científicas Sociales (NH: CCS). Marxismo- Fromm tiene como base las posturas del Marxismo (2011) y Fromm (1958, 1973), se compone por 9 afirmaciones de las 41 totales, donde se considera que el ser humano es efecto y causa de sus condiciones de vida, además de ser el resultado de procesos socio-culturales, por ello, sus necesidades son el reflejo de la sociedad. En este sentido, a las personas se les define a partir del momento histórico en el que se encuentran y la generación de conocimiento es lo que ha logrado hacerlos humanos. Su mayor valor absoluto es de .692 correspondiente al ítem "Pienso que el ser humano es autocreador de su contexto vital”. La varianza explicada de este factor representa un $10.420 \%$ con un valor de coeficiente Alpha de Cronbach de $\alpha=0.861$.

Al Factor 3 Origen de la Vida: Creencias Científicas Naturales (OV: $C C N)$. Evolución lo constituyen reactivos que hacen referencia a creencias cuya base sostiene que la Teoría de la Evolución de las Especies de Charles Darwin (Darwin, 2003) es la explicación más racional sobre el origen del hombre. Esta teoría, facilita la comprensión de la existencia de una multitud de especies, el proceso de evolución del que deviene el hombre, a través del estudio de fósiles, y la relevancia de la adaptación a un contexto natural. Con base en esta información se construyeron 6 afirmaciones de las 41 totales que conforman el instrumento. El mayor valor absoluto es de .698 correspondiente al ítem “. La similitud biológica entre diferentes seres vivos permite reconstruir la relación entre las diferentes líneas evolutivas”. La varianza explicada de este factor constituye un $8.623 \%$ con un valor de coeficiente Alpha de Cronbach de $\alpha=0.851$.

Finalmente el Factor 4 versa sobre la Naturaleza Humana: Creencias Científicas Sociales (NH: CCS) específicamente los postulados de E. Fromm. Los cuales sostienen que la cultura funge como creadora de lo humano, resaltando el papel protagónico de la interacción entre el hombre, su historia y la sociedad. Este factor se compone por 3 afirmaciones de las 41 totales. El mayor valor absoluto es de .636 correspondiente al ítem "Los 
seres humanos son una totalidad conformada por su familia y el resto de la sociedad”. La varianza explicada de este factor representa un $4.715 \%$ con un valor de coeficiente Alpha de Cronbach de $\alpha=0.529$.

\section{Correlación de Pearson}

Para conocer la relación existente entre los factores de estudio, su intensidad y su sentido se procedió a realizar la prueba de correlación de Pearson. Los resultados de esta prueba señalan que:

El Factor 1.NH-OV: CR interactúa significativamente con el Factor 2.NH-C.S. Marxismo-Fromm, con una correlación significativa Moderada con dirección negativa y un valor de coeficiente de -.428(**). Asimismo su interacción con el Factor 3.OV: CCN Evolución muestra una correlación Moderada con dirección negativa y un valor de coeficiente de -.507. En cuanto a su interacción con el Factor 4. NH: CCS. Fromm se encuentra una correlación significativa Baja con dirección positiva y un valor de coeficiente de $-.221(* *)$.

El Factor 2. NH: CCS. Marxismo- Fromm presenta una correlación significativa Alta con dirección positiva y un valor de coeficiente de .657(**) con el Factor 3.OV: CCN Evolución. De igual manera interactúa de forma significativa Moderada con dirección positiva y un valor de coeficiente de .588 con el Factor 4. NH:CCS. Fromm.

En cuanto a la interacción del Factor 3. OV: CCN. Con el Factor 4. $\mathrm{NH}$ :CCS. Fromm se muestra una correlación significativa Moderada con dirección positiva y un valor de coeficiente de .541(**) (Ver, Tabla 4).

Tabla 4. Análisis de correlación con el coeficiente r de Pearson

\begin{tabular}{|c|c|c|c|c|}
\hline & $\begin{array}{c}\text { Factor } 1 . \\
\text { NH-OV: } \\
\text { CR. } \\
\text { Religión }\end{array}$ & $\begin{array}{l}\text { Factor } 2 . \\
\text { NH: CCS. } \\
\text { Marxismo- } \\
\text { Fromm }\end{array}$ & $\begin{array}{l}\text { Factor } 3 . \\
\text { OV: CCN. } \\
\text { Evolución }\end{array}$ & $\begin{array}{c}\text { Factor } 4 . \\
\text { NH: CCS. } \\
\text { Fromm }\end{array}$ \\
\hline Factor 1. NH-OV: CR. Religión & 1 & & & \\
\hline $\begin{array}{c}\text { Factor 2. NH: CCS. Marxismo- } \\
\text { Fromm }\end{array}$ & $-.428^{* *}$ & 1 & & \\
\hline Factor 3. OV: CCN. Evolución & $-.507^{* *}$ & $.657^{* *}$ & 1 & \\
\hline Factor 4. NH: CCS. Fromm & $-.221^{* *}$ & $.588^{* *}$ & $.541^{* *}$ & 1 \\
\hline
\end{tabular}

Las correlaciones negativas encontradas entre el Factor 1. NH-OV: CR. Religión con el Factor 2. NH: CCS. Marxismo- Fromm, el Factor 3. OV: CCN. Evolución y el Factor 4. NH:CCS. Fromm indican una incompatibilidad de creencias religiosas y creencias científicas tanto sociales como naturales. Entendiéndose que la población en general que se inclina por explicaciones de corte religioso ante el cuestionamiento sobre el origen de la vida y la naturaleza humana soportan sus argumentos tanto en la concepción de una verdad eterna y cierta del mundo como se conoce; como 
de los libros y escrituras sagradas, a los cuales los mantienen intactos y los aceptan como un todo (Russell, 2012). Retomando así dentro del marco de la religión la creencia en Dios, el alma y la inmortalidad (Leuba, 1921; Nature Editorial, 2005); la creencia de que el destino humano es la fe en un Dios personal (Tenney, 1982, citado en, Kuwornu-Adjaottor, 2013), así como, en la voluntad, la personalidad y la expresión de un ser trascendente para conocer el mundo (Pinker, 2003; Dembski, 2005, 2007).

Para el Factor 2NH: CCS. Marxismo-Fromm los resultados muestran una correlación estadísticamente significativa con una dirección positiva con los factores 3. OV: CCN. Evolución y el 4 NH: CCS. Fromm, lo cual significa la población en general que cree en teorías científicas sociales basadas en las posturas del Materialismo Dialéctico de Marx y Engels (2011) coinciden con posturas científicas naturales y sociales como la de Fromm. En este contexto la población con una visión científica social que concibe la naturaleza del hombre inherente a su historia de vida (Marx y Engels ,2011) y reafirma la relevancia del entorno social (Radhakrishnan, 1976; Ritzer, 2005) acepta también explicaciones proporcionadas por la Teoría de la Evolución de las Especies de Charles Darwin (Darwin, 2003) y la postura de Fromm (1958,1973). Esto sin necesidad de apelar a un ente creador o diseñador, debido a que, ésta reposa sobre unos cimientos sólidos formados por observación, experimentación y evidencias que confirman su validez.

Así mismo siendo el Factor 3. OV: CCN. Evolución y el factor 4. NH: CCS. Fromm pertenecientes a la vertiente Científica se encontró una correlación positiva, lo cual demuestra una coexistencia entre creencias científicas sociales de Fromm $(1958,1973)$ y creencias naturales propiamente sustentadas por la Teoría de la Evolución de las Especies de Charles Darwin (Darwin, 2003); al explicar la naturaleza y origen del hombre.

\section{t de Student para muestras independientes}

Con el objetivo de conocer si existe una diferencia significativa según el sexo se realizó un análisis de t de Student para muestras independientes en cada uno de los factores presentados.

Los resultados indican que únicamente existen diferencias estadísticamente significativas en la variable sexo dentro del Factor 1 . NHOV: CR. Religión ( $t=3.014$, gl=598, $\mathrm{p}<.003)$.Las mujeres $(\mathrm{M}=2.73)$ creen que la vida en la Tierra debió ser el resultado de una obra divina dirigida por un ser superior, incluyendo la actual compleja estructura biológica de los seres vivos, es decir, apoyan las posturas religiosas integradas por el Creacionismo (Gould, 2000,2006), el Judeocristianismo (Pinker, 2003) y la Teoría del Diseño Inteligente (Dembski, 2005, 2007). 
En cuanto a los hombres $(M=3.02)$ se inclinan más hacia posturas científicas tanto sociales como naturales tales como la Teoría de la Evolución de Charles Darwin (Darwin, 2003); el Materialismo Dialéctico de Marx y Engels (2011) y los postulados de Fromm (1958, 1973), para darle explicación a los cuestionamientos referentes al origen y naturaleza humana. Esto significa que están de acuerdo con las explicaciones que permiten mayor control y predicción sobre los fenómenos naturales, sociales y culturales (Pérez, Gutiérrez, García, Gómez, 2005).

Estos resultados indican claramente que existen diferencias estadísticamente significativas entre las creencias hacia el origen de la vida y la naturaleza humana entre hombres y mujeres.

\section{Análisis de Varianza (ANOVAS)}

Con el fin de conocer si existen diferencias estadísticamente significativas en la muestra y las variables: formación académica, edad y el estado civil se realizó un análisis de varianza.

Los resultados muestran que existen diferencias estadísticamente significativas entre el Factor 1. NH-OV: CR. Religión $(F=75.877, g l=4$, $p<.000$ ) y la escolaridad. Siendo las personas que cuentan con un nivel académico de primaria $(M=1.82)$ quienes optan por explicaciones de corte religioso sustentadas en creencias que dictan la voluntad, la personalidad y la expresión de las intenciones de un ser trascendente son causa directa del origen y naturaleza humana; en contraste con las personas que cuentan con un grado de estudios de Licenciatura $(M=3.53)$, observando que la diferencia mayor encontrada entre medias es de 1.71 unidades.

En cuanto al Factor 2NH: CCS. Marxismo-Fromm $(F=34.049, g l=4$, $p<.000$ ) la diferencia mayor registrada entre las medias es de 0.56 unidades entre las personas con grado académico de Licenciatura $(\mathrm{M}=1.39)$ y quienes estudiaron la preparatoria ( $\mathrm{M}=1.95)$.

Para el Factor 3. OV: CCN. Evolución $(F=41.486, g l=4, p<.000)$ se encontró que los sujetos que contaban con un posgrado $(M=2.02)$ muestran mayor inclinación hacia las explicaciones basadas en la Teoría de la Evolución para explicar el origen de la vida y la naturaleza humana a diferencia de quienes contaban con la preparatoria $(\mathrm{M}=3.08)$, existiendo entre estas una diferencia de mediad de 1.06 unidades.

El Factor 4. NH: CCS. Fromm $(F=16.412, g l=4, p<.000)$ cuenta con una diferencia de medias de .66 unidades entre las personas con posgrado $(\mathrm{M}=2.18)$ y las que tienen un grado de secundaria $(\mathrm{M}=2.84)$.

Los datos significativos en los cuatro factores demuestran la relevancia que puede tener la escolaridad como variable predictiva de las creencias. 
La información registrada para la variable edad muestra que hay diferencias significativas en tres de los factores:

Factor 1. NH-OV: CR. Religión ( $\mathrm{F}=4.951, \mathrm{gl}=3, \mathrm{p}<.002)$ registra una diferencia de medias de 0.6 unidades correspondiendo la mayor a las personas mayores de 50 años $(M=3.08)$ y la menor al sector de entre 20 y 29 años $(\mathrm{M}=2.48)$, lo cual indica que estos últimos tienen predilección por las creencias religiosas.

El Factor 3. OV: CCN. Evolución $(\mathrm{F}=3.974, \mathrm{gl}=3, \mathrm{p}<.008)$ presentó una diferencia de medias de 0.23 unidades entre las categorías de 20 a 29 años $(\mathrm{M}=2.51)$ y los mayores de 50 años $(\mathrm{M}=2.25)$, que se interpreta como mayor apoyo de la población de 50 años en adelante hacia las creencias científicas de corte natural.

Así mismo el Factor 4. NH: CCS. Fromm ( $\mathrm{F}=6.902$, gl=3, $\mathrm{p}<.000)$ conformado por los postulados de Fromm $(1958,1973)$ y parte fundamental de las creencias sociales que explican el origen de la vida y la naturaleza humana, constituye la postura hacia las que se inclinan los adultos de más de 50 años $(M=2.25)$ en contraste con las personas que se encuentran en el rango de edad de entre 20 a 29 años $(M=2.78)$, encontrándose entre estos la mayor diferencia de medias con un valor de 0.53 .

En conjunto los datos relacionados con la variable edad muestran una mayor inclinación de las personas mayores de 50 años hacia las teorías científicas tanto sociales como naturales acompañada de una disminución de creencias religiosas. Por el contrario el grupo de menor edad, conformado por personas de 20 a 29 años, mantiene una visión mayormente religiosa.

La variable estado civil presenta una relación estadísticamente significativa con la muestra estudiada únicamente en el Factor 1. NH-OV: CR. Religión ( $\mathrm{F}=6.02$, gl=2, $\mathrm{p}<.003$ ) con una diferencia mayor de medias de 0.72 unidades entre la categoría Unión Libre $(\mathrm{M}=2.52)$ y solteros $(\mathrm{M}=3.04)$, entendiéndose que las personas que viven juntos sin estar casados creen en mayor medida en un ser superior como Creador o Diseñador de la vida y naturaleza humana.

\section{Conclusión}

Las creencias son el eje que permite dar sentido a la realidad, la información que las personas conservan sobre un objeto o fenómeno no necesariamente tiene que ser verdadera para ser admitida como real. Las personas pueden guardar una imagen de la realidad sin tener fundamento científico alguno que justifique sus creencias; del mismo modo, pueden enfrentar argumentos que resulten ser contrarios a su concepción, y aun así, continuar creyendo. Las creencias son aceptadas como algo real, en la medida que se fundan en la existencia de los hombres y sirven como fundamento de la vida. De ahí que el gran abanico de creencias que poseen 
las personas es el que les brinda el marco de referencia que determina su forma de ser en el mundo, el modo en que evalúa las situaciones, a los demás, a sí mismo, la forma en que interactúa con su entorno e influye en su pensamiento condicionando su toma de decisiones. En este sentido, la creencia actúa como disposición a la acción, como el mayor determinante del comportamiento humano (Beck, 1976, citados en, Calvete y Cardeñoso, 2001; Brown \& Cooney, 1982; Chan \& Elliott, 2004; Bandura, 1986; Dweck, 1999; Hofer, 2004, citados en, García y Sebastián, 2011; Dewy, 1984; Sigel, 1985, citados en, Latorre y Blanco, 2007; Fishbein y Ajzen, 1975; Olson y Zanna, 1987; Pajares, 1992; Ponte, 1994; Richards y Lockhart, 1994, citados en, Mohamed, 2006; Rokeach, 1968, citados en, Pajares, 1992; Villoro, 1996).

Bajo este contexto, las creencias científicas de orden natural y social respecto al origen de la vida y naturaleza humana que mantienen la población en general, tienen como base teorías científicas que se fundamentan en una serie de ideas racionales que les permite generar conocimientos objetivos y trabajar con determinados procesos metodológicos que se adscriben a modelos empíricos orientados a la comprobación de hipótesis mediante métodos, teorías y leyes. Mientras tanto, las creencias religiosas que presenta una porción las personas de la población en general, también tienen principios que se fundamentan dentro de un orden cultural en tanto se construyen dentro de la interacción social (Nespor, 1987; Pajares, 1992; Park, 2012; Pepitone, 1991; Solar y Díaz, 2009). La presencia de estas creencias religiosas, muestra la gran la influencia que las diferentes corrientes religiosas han tenido en el ser humano, casi siempre debido a su escaso conocimiento de la dinámica socio cultural y el papel que esta juega en la determinación de las creencias, sobre todo cuando grandes sectores de la población tienen escaso acceso a la educación, o su educación es básica, no permitiéndoles el enjuiciamiento o confrontación de sus creencias de sentido común con la realidad, dado que, carecen de elementos teórico metodológicos y científicos que les posibilite entender la conformación de la realidad, y de esta forma cambiar sus creencias de sentido común por otras con fundamento científico.

Las personas asumen en su vida familiar, social y laboral, etc., una versión de los fenómenos de la realidad, por ello, sus creencias pueden verse como un substrato conceptual que juega un papel importante en su pensamiento y acción, influyen en ocasiones de manera determinante en las diferentes esferas de la vida (familiar, social y laboral, etc.); siendo la función de las creencias, en estas esferas la de actuar como filtro de entrada de información que sugiere importantes implicaciones en el desarrollo y formación de las personas (Mohamed, 2006; Ponte, 1994; Thompson, 1992). 
Como consecuencia de lo anterior, se puede encontrar una gran variedad de personas, es decir hombres, mujeres, solteros ,casados, de una u otra edad, con educación básica o con estudios profesionales cuya labor depende de los intereses y contextos personales y sociales, los cuales a su vez, se encuentran sujetos a condiciones económicas, políticas e institucionales; pero al final, sus creencias permean y opacan a los avances del conocimiento el cual les permitiría tener una mejor explicación a diferentes fenómenos del Universo, del mundo, de la realidad y del propio origen y naturaleza del ser humano.

En términos generales, las creencias sobre el origen de la vida y naturaleza humana que plantean tanto los principios científicos como los religiosos a menudo difieren. Esta diferencia ha traído consecuencias en las decisiones y acciones del ser humano, donde muchas veces éste se ha visto obligado a elegir una versión de la realidad (Scheitle, 2011).

Ante esto, conocer estas creencias en población en general proporciona una visión integral de su comportamiento en todas las esferas de su vida (Nespor, 1987).

El papel que desempeñan las creencias en el comportamiento de las

personas se caracteriza básicamente por el cómo se adquiere el conocimiento base de su teoría y práctica. De ahí que el gran abanico de creencias que poseen las personas es el que les brinda el marco de referencia que determina los procesos y principios científicos y no científicos que estructuran su interacción con la realidad, con el mundo, con sus iguales y consigo mismo.

\section{References:}

1. Arispe, L. (1989). Cultura y desarrollo. Una etnografía de las creencias de una comunidad mexicana. México: UNAM-Porrúa.

2. Calvete, E. y Cardeñoso, O. (2001). Creencias, resolución de problemas sociales y correlatos psicológicos. Psicothema, 13 (1), 95100.

3. Darwin, Ch. (1987). El Origen del Hombre (1 $1^{\mathrm{a}}$. Reimpresión). México: Editores Unidos.

4. Darwin, Ch. (2003). El Origen de las especies. Madrid. Alianza

5. Dembski, W. (2005). Diseño Inteligente. Un puente entre ciencia y la tecnología. Miami: Vida.

6. Dembski, W. (2007). En defensa del Diseño Inteligente. Praxis Filosófica, 24, 147-166.

7. Díaz, L. (2004). Por qué creemos lo que creemos: un enfoque psicosocial. En I. Rodríguez (Colaborador), Ciencia y pseudociencia: realidades y mitos (pp. 339-349). Madrid: Equipo Sirius. 
8. Fishbein, M. \& Ajzen, I. (1975). Belief, Attitude, Intention, and Behavior: An Introduction to Theory and Research. Reading, MA: Addison-Wesley.

9. Fishbein, M. (1967). A consideration of beliefs and their role in attitude measurement. In, M. Fishbein (Ed.), Readings in attitude theory and measurement (pp. 257-266). New York: John Wiley and Sons.

10. Fromm, E. (1958). Psicoanálisis de la sociedad contemporánea (6 ed.). México: Fondo de Cultura Económica.

11. Fromm, E. (1973). Marx y su concepto del hombre (5a Reimpresión). México: Fondo de Cultura Económica.

12. García, J. (2009). Caminos de la reflexión La teoría Orteguiana de las ideas y las creencias en el contexto de la escuela de Madrid. España: Biblioteca Nueva

13. García, M. y Sebastián, C. (2011). Creencias Epistemológicas de Estudiantes de Pedagogía en Educación Parvularia, Básica y Media: ¿Diferencias en la Formación Inicial Docente? PSYKHE, 20 (1), 2943.

14. Gould, S. (2000).Ciencia versus religión. Un falso conflicto. Barcelona: Crítica.

15. Gould, S. (2006). Ocho cerditos. Reflexiones sobre historia natural. Barcelona: Critica.

16. Kuwornu-Adjaottor, J. (2013). 'You believe in God' and 'Believe in God': a critical study of John 14:1.1st Annual International Interdisciplinary Conference, AIIC. University of The Azores and the European Scientific Institute, ESI. Azores, Portugal.

17. Latorre, M. y Blanco, F. (2007). Algunos conceptos clave en torno a las creencias de los docentes en formación. Docencia e Investigación. Revista de la Escuela Universitaria de Magisterio de Toledo, 7, 147170.

18. Leuba, J. (1921). The belief in God and Immortality. Estados Unidos: The Open Court Publishing Company.

19. Llinares, S. (1995). Conocimiento profesional del profesor de matemáticas: Conocimiento, creencias y contexto en relación a la noción de función. Conferencia invitada en el IV Encuentro de Investigación en Educación Matemática. Luso. Portugal.

20. Mohamed, N. (2006). An Exploratory Study of the Interplay between Teachers' Beliefs, Instructional Practices \& Professional Development. Tesis de Doctorado para la obtención del título de Doctor en Filosofía en la enseñanza y aprendizaje. Universidad de Auckland, Nueva Zelanda.

21. Nature Editorial. (2005). Dealing with design. Nature, 434, 1053. 
22. Nespor, J. (1987). The role of beliefs in the practice of teaching. Journal of Curriculum Studies, 19 (4), 317-328.

23. Olson, J. y Zanna, M. (1987). Actitudes y Creencias. En, Daniel Perlman y P. Cosby (Eds.), Psicología Social (pp. 71-91). México: Trillas.

24. Pajares, F. (1992). Teachers' beliefs and educational research: cleaning up a messy construct. Review of Educational Research, 62 (3), 307-332.

25. Park, C. (2012, Agosto). Attending to the construct of beliefs in research on religion/ spirituality and health: Commentary on 'Beyond belief'. Journal of Health Psychology, 17 (7), 969 -973.

26. Pepitone, A. (1991). El mundo de las creencias: un análisis psicosocial. Revista de Psicología Social y Personalidad, 7 (1), 6179.

27. Pérez, Vicente; Gutiérrez, María; García, Andrés y Gómez, Jesús. (2005). Procesos Psicológicos Básicos. Un análisis Funcional. España: Pearson. Pretince Hall.

28. Pinker, S. (2003). La Tabla Rasa. La negación moderna de la naturaleza humana. Barcelona: Paidos Transiciones.

29. Ponte, J. (1994). Knowledge, beliefs and conceptions in mathematics teaching and learning. In, L. Bazzini (Ed.), Theory and practice in mathematics education. Proceedings of the Fifth international conference on systematic cooperation between theory and practice in mathematics education. Italia: Grado.

30. Radhakrishnan, S. (1976). El concepto del hombre: Estudio de Filosofía. México: Fondo de Cultura Económica.

31. Ritzer, G. (2005). Teoría Sociológica Clásica. México: Mc Graw Hill.

32. Rodríguez, F. y Sotelo, G. (2007). Creencias ambientales en estudiantes de la UNAM. Tesis de licenciatura. Facultad de Estudios Superiores Zaragoza, UNAM.

33. Russell, B. (2012). Religión y Ciencia. México: Fondo de Cultura Económica.

34. Scheitle, Ch. (2011). U.S. College Students' Perception of Religion and Science: Conflict, Collaboration, or Independence? A Research Note. Journal for the Scientific Study of Religion, 50 (1), 175-186.

35. Solar, M. y Díaz, C. (2009). El profesor universitario: construcción de su saber pedagógico e identidad profesional a partir de sus cogniciones y creencias. Revista Calidad en la Educación, 30, 207232. 
36. Thompson, A. (1992). Teacher's beliefs and conceptions: a synthesis of the research. In, D. Grouws (Ed.), Handbook on mathematics teaching and learning (pp.127-146). New York: Macmillan.

37. Villoro, L. (1996). Creer, saber, conocer (9a. Ed.). México: Siglo XXI. 